\title{
SOCIAL MEDIA RISK MANAGEMENT STRATEGY - APPLYING THE ANALYTIC HIERARCHY PROCESS
}

\author{
Kanwal Rai \\ Capgemini India Private Limited \\ Crescent 2, Prestige Shantiniketan \\ Sadaramangala Village, Whitefield Main Road \\ Bangalore - 560 048, INDIA \\ E-mail: kanwal.rai@capgemini.com \\ Navneet Bhushan \\ Crafitti Consulting Private Limited, \\ 1B-401, Akme Harmony, \\ Sarjapur Outer Ring Road, Bellandur, \\ Bangalore - 560103, INDIA \\ E-mail: navneet.bhushan@crafitti.com
}

\begin{abstract}
Social Media is an ever emerging dynamic and complex ecosystem of people and communication process supported by diversified set of underlying digital technology platforms. Technology assisted production, propagation and consumption of information process is intrinsically governed by the intent of the people embedded within a context which is a time variant influencing or masking factor. The complexity and impact of this information system grows exponentially as it tends to become vibrant and volatile with multiplicity of choices for content, channels and active communities on a social network. Now a days it is being observed that such an emotional vibrancy in the digital network is reaching a tipping point quite often, wherein an impact, positive or negative, spills beyond the social limits of the technology platforms, and paves its path into the physical world with far reaching repercussions, almost on instant basis, without any respect for the geographical measures of control and containment. There are no set predefined guidelines or obligations that can be imposed on it as levers to fully control the risks associated with the negative impacts, as they are shepherd by the diversified set of spontaneous perspectives and intents embedded within a sensitivity of a context. In short, an effective and targeted response to control or eliminate the risk or disaster originating from these social media platforms, can be designed and executed only after a thorough analysis of impacts, under consideration.
\end{abstract}

Although a specific risk response is to be designed to address a specific negative impact, present under a given context, pre-acquired knowledge of meta-characteristics of various dimensions of social media system behaviour like intent, content, channel, context and impact would help in selecting the right strategy for a risk response. The main objective of this paper is to systematically structure and analyze the various characteristics of the above mentioned dimensions of social media and provide an AHP based framework to compute their significance in order to support the risk management team in designing the best possible risk response strategy for a particular type of an impact.

Key words: Social Media, Social Media Risk, Risk Management, Strategy, Social Media Platforms 
ISAHP 2014: Rai, K \& Bhushan, N/ Social Media Risk Management Strategy - Applying the Analytic Hierarchy Process submitted to the International Symposium of the Analytic Hierarchy Process 2014, Washington D.C., U.S.A

*Corresponding Author: kanwal.rai@,capgemini.com Ph: +91 9886003499, navneet.bhushan@crafitti.com Ph: +91 9902766961 